\title{
ГОЛОМОРФНОЕ ПОГРУЖЕНИЕ КАК АНАЛИТИЧЕСКИЙ МЕТОД РАСЧЕТА ЭЛЕКТРИЧЕСКИХ СЕТЕЙ НЕФТЯНЫХ И ГАЗОВЫХ МЕСТОРОЖДЕНИЙ
}

\author{
Исаев Юсуп Ниязбекович ${ }^{1}$, \\ isaev_yusup@mail.ru \\ Кабалин Дмитрий Андреевич1, \\ mitya.kabalin@gmail.com \\ 1 Национальный исследовательский Томский политехнический университет, \\ Россия, 634050, г. Томск, пр. Ленина, 30.
}

\begin{abstract}
Для того чтобы обеспечить требуемый уровень надежного функционирования объектов нефтедобычи, необходимо обращать внимание на условия эксплуатации энергосистемы. Это важно, если принять во внимание, что для добычи несрти и газа необходимы достаточно мощные источники энергии, которые существенно влияют на режим работы электросети. При этом актуальным является задача расчета установившихся режимов электрической сети, питающей объекты нефттедобычи. Расчеты установившихся режимов имеют большое практическое значение для обеспечения эффрективного и безопасного управления режимами работы нефтяных и газовых предприятий, являются важными при проектировании электрических сетей, питающих объекты нефтегазовых предприятий. Однако применение классических итерационных методов расчета установившихся режимов, таких как метод Гаусса-Зайделя и Ньютона-Рафсона, не всегда позволяет найти правильное решение системы нелинейных уравнений, описывающих установившиеся режимы работы сети, так как сходимость данных методов зависит от начальных приближений. В работе предлагается аналитический метод расчета установившихся режимов электрических сетей, описываемых нелинейными уравнениями. Метод основан на аппроксимации Паде и методе возмущений. Приводятся преимущества метода перед известным методом итераций Гаусса-Зайделя и Ньютона-Рафрсона. Приводятся примеры решения задач электроэнергетических сетей, и обсуждаются недостатки предлагаемого метода. Рассматриваются проблемы устойчивости.

Цель: применить аналитический метод голоморфного погружения для расчета двух и трехузловой энергетической схемы; сравнить возможности метода с другими альтернативными методами; исследовать ограничения метода голоморфного погружения и показать область его работы.
\end{abstract}

Методы: разложение Тейлора, аналитическое продолжение, решения алгебраических уравнений рекуррентным методом, бесконечные дроби.

Результаты. Рассмотрены примеры использования метода голоморфного погружения для двух и трех $P Q$ узловых схем, и показаны недостатки метода голоморфного погружения. Проведено сравнение метода голоморфного погружения с альтернативными методами.

Выводы. Аналитический метод голоморфного погружения обладает рядом преимуществ: физической наглядностью, простотой алгоритмической реализации, заключающейся в рекуррентных соотношениях для коэффрициентов разложения искомой фуннкции в ряд Тейлора. Разложенная в ряд функция является голоморфной, что позволяет осуществлять ее аналитическое продолжение и получить желаемую точность решения.

\section{Ключевые слова:}

Энергосистема, установившийся процесс, устойчивость, нелинейные уравнения, голоморфное погружение, разложение Паде, сходимость.

\section{Введение}

Для добычи нефти и газа необходимы достаточно мощные источники энергии. В связи с этим возникают вопросы допустимых электроэнергетических режимов работы нефтяных и газовых месторождений. Для того чтобы обеспечить требуемый уровень надежного функционирования процессов нефтедобычи, необходимо обращать внимание на условия эксплуатации энергосистемы. При этом актуальной является задача расчета установившихся режимов (УР) электрической сети, питающей объекты нефтедобычи. Расчеты установившихся режимов имеют большое практическое значение для обеспечения эффективного и безопасного управления режимами работы нефтяных и газовых предприятий, являются важными при проектировании электрических сетей для нефтегазовых предприятий. Наряду с расчетами УР важными являются вопросы исследования устойчивости работы энергосети. Нарушения устойчивости приво- дят к торможению и отключению электроприемников объектов нефтегазовой отрасли [1-4]. Поэтому работа посвящена расчету УР электрических сетей и вопросам устойчивости функционирования энергосети.

Особенности электроэнергетических систем заключаются в том, что расчет УР производится с помощью нелинейных алгебраических уравнений. Для решения нелинейных уравнений применяют приближенные итерационные методы, позволяющие определить решение системы уравнений с заданной точностью [3-7]. В течение многих десятилетий для решения систем нелинейных алгебраических уравнений использовались такие итерационные методы, как метод Гаусса-Зайделя и метод Ньютона-Рафсона. Метод Гаусса-Зайделя прост в реализации и требует мало памяти ЭВМ, но имеет неудовлетворительную сходимость к решению. Метод Ньютона-Рафсона обладает на порядок лучшей сходимостью (квадратичной), однако при этом в вычислительном отноше- 
нии он требует большего объема памяти ЭВМ [3-7]. Существует также множество модифицированных методов, основу которых составляют вышеперечисленные методы. Наибольшее распространение получил метод Ньютона-Рафсона, который применяется в большинстве современных программных комплексов расчета режимов, как отечественных, так и зарубежных.

Хотя итерационные методы широко применяются, у них имеются недостатки. В частности, итерационный процесс зависит от начальных приближений, при этом итерационный процесс может расходиться, а также возможна сходимость к решениям, не имеющим физического смысла. Кроме того, одной из основных проблем является возможное расхождение итерационного процесс, даже если решение существует. Данная проблема часто встречается при расчете режимов, близких к предельным [3-7].

С интенсивным развитием математики последнее время большое внимание уделяется аналитическим методам решениям нелинейных задач, в частности для их решения был предложен неитерационный метод голоморфного погружения (ГП), основанный на комплексном анализе. Важной особенностью данного метода является однозначность решения: метод теоретически гарантированно сходится к решению независимо от начального приближения. Первым на возможность аналитического решения обратил внимание испанский исследователь Антонио Триас (Antonio Trias) [8]. Им было предложено использовать метод ГП для решения задачи расчета режимов электроэнергетических систем. Метод обладает наглядностью анализа происходящих процессов, простотой и изяществом.

\section{Описание метода голоморфного погружения}

Суть метода заключается в том, что в нелинейное уравнение вводится дополнительная комплексная координата - параметр погружения $\alpha$, позволяющая преобразовать искомое решение в голоморфную функцию [9]. Искомое решение разлагается в ряд Тейлора по параметру погружения $\alpha$. В этой части метод ГП напоминает известный метод разложения решения по малому параметру возмущения [10]. В зависимости от желаемой точности удерживается соответствующее число членов разложения. Поскольку полученное решение является голоморфной функцией, то можно осуществить ее аналитическое продолжение, используя аппроксимацию Паде по параметру $\alpha[11-14]$. При этом увеличивается радиус сходимости искомого решения, что позволяет улучшить желаемую точность решения. Для получения искомого решения вместо параметра погружения $\alpha$ нужно подставить единицу. Простота метода заключается и в том, что нелинейное уравнение заменяется системой линейных рекуррентных уравнений, что легко реализуется алгоритмически. Как показано в $[8,14]$, важной особенностью метода ГП является однозначность решения: «если решение существует, то это соответствует фактическому состоянию работы энергосистемы, если решения нет, значит, в системе лавина напряжения» [8].

\section{Устойчивость двухузловой энергосистемы}

Чтобы судить об эффективности работы того или иного метода расчета энергосистемы, необходимо сформулировать соответствующие критерии. В этом разделе рассмотрим вопросы устойчивости на примере энергосистемы с двумя узлами и приведем пример решения задачи аналитическим методом. Метод наглядно показывает границу устойчивости, области устойчивости и неустойчивости. При увеличении мощности нагрузки, при фиксированном значении напряжения шины генератора и сопротивления связи, происходит утяжеление режима работы энергосистемы. Утяжеления можно добиться и увеличением сопротивления связи. В математическом смысле утяжеление режима работы энергосистемы означает приближение решения нелинейного уравнения, описывающего энергосистему, к границе устойчивости. Если решения находятся за границей устойчивости, это значит, что в энергосистеме происходит лавина напряжения и, следовательно, решения не имеют физического смысла, однако в математическом смысле они существуют. Аналитический метод хорош тем, что решения, полученные этим методом, могут быть использованы для верификации альтернативных методов расчета и позволяют явно демонстрировать их преимущества и недостатки. В частности, при приближении к границе устойчивости метод НьютонаРафсона решение начинает расходиться. А метод ГП выдает некорректные решения из-за наличия ложных нулей в знаменателе Паде разложения.

В приведенной схеме (рис. 1) величины $Z$ - сопротивление связи, $S$ - мощность, потребляемая нагрузкой, $U_{1}$ - напряжение на шинах генератора известны, нужно определить $V$.

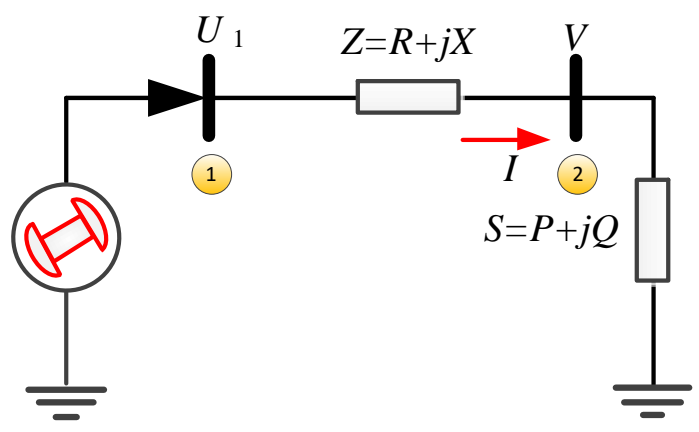

Рис. 1. Двухузловая однолинейная схема электропередачи Fig. 1. Two bus one-line transmission system diagram

Запишем уравнение для определения неизвестного напряжения $V$, предполагая, что напряжение для $U_{1}$ является действительной величиной.

Мощность в узле 2 определяется выражением $S=I^{*} V$. Где * - означает комплексное сопряжение. Проделав определенную последовательность действий, получим уравнение для определения напряжения в узле 2

$$
S=I^{*} V \rightarrow I=\frac{U_{1}-V}{Z} \rightarrow \frac{U_{1}-V^{*}}{Z^{*}} V=P+j Q .
$$


Упростив выражение, можно получить квадратное уравнения относительно неизвестной величины $V$ :

$$
\begin{gathered}
\left(U_{1}-V^{*}\right) V=(P+j Q)(R-j X) \rightarrow-V^{2}+V U_{1}= \\
=(P R+Q X)+j(Q R-P X)
\end{gathered}
$$

или

$$
\left(V_{r}^{2}+V_{i}^{2}\right)-V U_{1}=-(P R+Q X)-j(Q R-P X) .
$$

И, наконец, получаем, изменяя все знаки и учитывая, что $V=V_{\mathrm{r}}+j V_{\mathrm{i}}$

$$
\begin{gathered}
\left(V_{r}^{2}+V_{i}^{2}\right)-U_{1}\left(V_{r}+j V_{i}\right)= \\
=-(P R+Q X)-j(Q R-P X) .
\end{gathered}
$$

Из последнего уравнения получаем систему уравнений, введя новые обозначения

$$
\begin{gathered}
\left\{\begin{array}{l}
\sigma_{r}=-(P R+Q X), \sigma_{i}=-(Q R-P X) \\
\left(V_{r}^{2}+V_{i}^{2}\right)-U_{1}\left(V_{r}+j V_{i}\right)=\sigma_{r}+j \sigma_{i} .
\end{array} \rightarrow\right. \\
\rightarrow\left\{\begin{array}{l}
V_{r}^{2}+V_{i}^{2}-U_{1} V_{r}=\sigma_{r} \\
V_{i}=-\frac{\sigma_{i}}{U_{1}} \rightarrow V_{r}^{2}-U_{1} V_{r}+\left(\sigma_{i}^{2}-\sigma_{r}\right)=0 .
\end{array}\right.
\end{gathered}
$$

Для действительной части искомого напряжения $V_{r}$ получаем решение, учитывая, что напряжение генератора в относительных единицах $U_{1}=1$,

$$
\begin{gathered}
V_{r}^{2}-V_{r}+\left(\sigma_{i}^{2}-\sigma_{r}\right)=0, \quad D=1-4\left(\sigma_{i}^{2}-\sigma_{r}\right) ; \\
V_{r}=\frac{1}{2} \pm \sqrt{\frac{D}{4}}=\frac{1}{2} \pm \sqrt{\frac{1}{4}+\sigma_{r}-\sigma_{i}^{2}} .
\end{gathered}
$$

С учетом его мнимой части можно получить выражение для комплексной величины $V=V_{r}+j V_{i}$

$$
V=V_{r}+j V_{i}=\frac{1}{2} \pm \sqrt{\frac{1}{4}+\sigma_{r}-\sigma_{i}^{2}}+j \sigma_{i} .
$$

Очевидно, что множество возможных решений определяется подкоренным выражением

$$
\frac{1}{4}+\sigma_{r}-\sigma_{i}^{2}=0, \text { или } \sigma_{r}=\sigma_{i}^{2}-\frac{1}{4} .
$$

Графически это можно представить в виде параболы. На левом графике рис. 2 приведены области, разделенные параболой.

Область существующего физического или устойчивого решения - точки, которые находятся в пределах параболы. Область несуществующего действительного решения (нефизические решения), неустойчивые решения, - точки за пределами параболы. Точки, находящиеся на параболе, дают корни, находящиеся на границе устойчивости, - этот случай соответствует двум одинаковым корням. На правом графике приведено соответствие между границей устойчивости и корнями действительной части напряжения $V_{r}$.
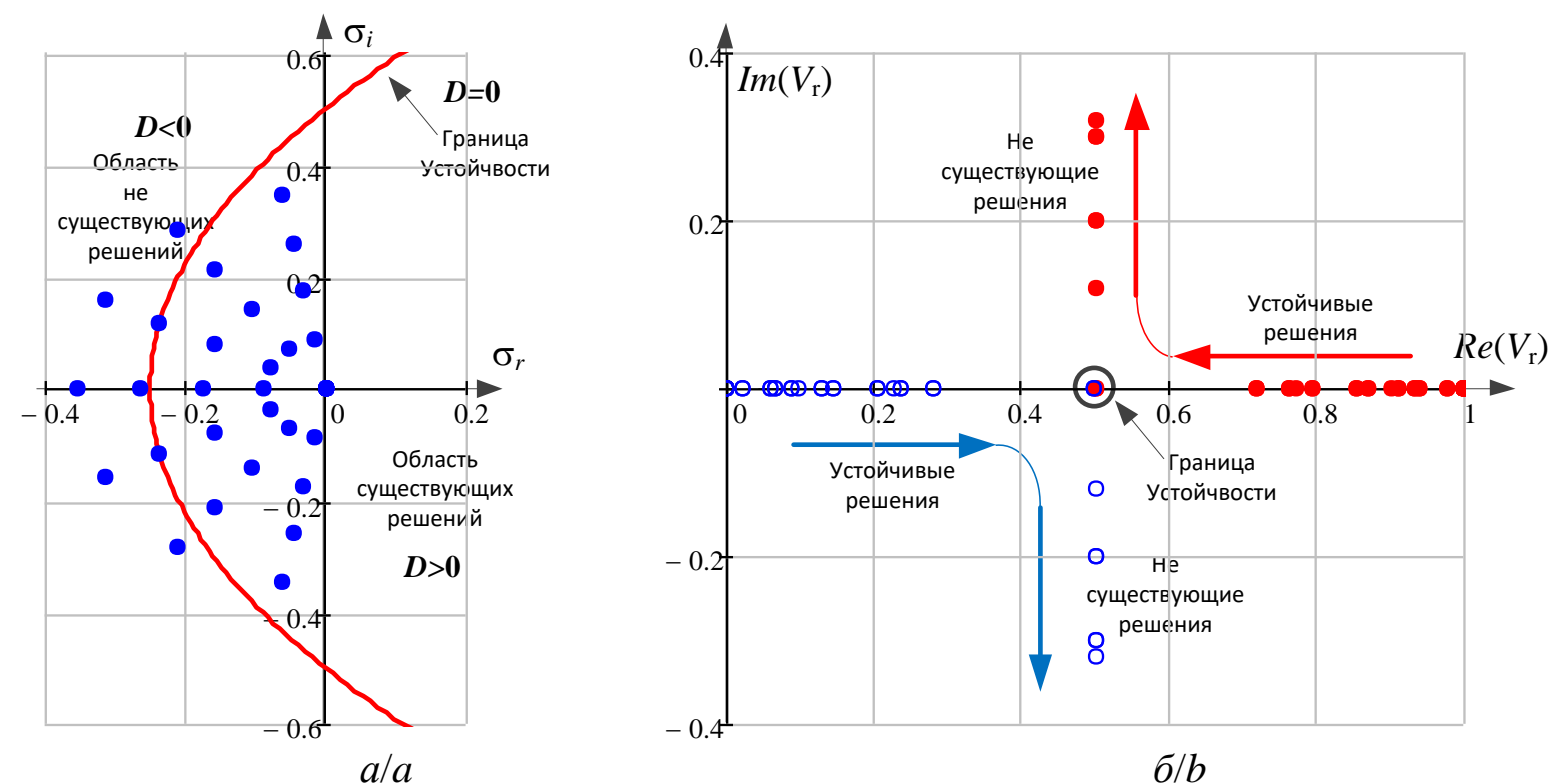

Рис. 2. Картина расположения корней по отнотению к границе устойчивости: а) разделения области на устойчивую и неустойчивую подобласти; б) годограф корней действительной части напряжения по отношению $к$ граниие устойчивости

Fig. 2. Picture of roots location with respect to the stability boundary: a) division of the region into a stable and unstable subdomains; b) real part of voltage locus with respect to the stability boundary

Пример использования метода голоморфного погружения для двухузловой схемы

Приведем пример использования метода ГП для двухузловой схемы, представленной на рис. 1, в которой требуется определить напряжения шины $V$ с нагрузкой, потребляющей мощность $S=P+j Q$, при условии, что напряжение узла генератора известно $U_{1}=1$.

Запишем полную мощность для нагрузки через ток и искомое напряжение и приведем решение методом голоморфного погружения. 


$$
\begin{gathered}
S=I^{*} V \rightarrow I=\frac{U_{1}-V}{Z} \rightarrow \frac{U_{1}-V}{Z}=\frac{S^{*}}{V^{*}} ; \\
V=U_{1}-\frac{S^{*} Z}{V^{*}} .
\end{gathered}
$$

Учитывая, что напряжение генератора в относительных единицах $U_{1}=1$, получаем

$$
V=1+\frac{\sigma}{V^{*}}, \sigma_{r}=-(P R+Q X), \sigma_{i}=-(Q R-P X) .
$$

Осуществим голоморфное погружение уравнения по параметру $\alpha$

$$
V(\alpha)=1+\frac{\alpha \sigma}{V^{*}(\alpha)} .
$$

Будем искать решения в виде разложения в ряд Тейлора

$$
\begin{aligned}
& V(\alpha)=1+c_{1} \alpha+c_{2} \alpha^{2}+c_{3} \alpha^{3}+c_{4} \alpha^{4}+\ldots \ldots \\
& \frac{1}{V(\alpha)}=1+d_{1} \alpha+d_{2} \alpha_{2}+d_{3} \alpha^{3}+d_{4} \alpha^{4}+\ldots \ldots
\end{aligned}
$$

Первые коэффициенты разложения определяются из соотношения

$$
V(0) / V(0)=1, d_{0}=1, c_{0}=1 .
$$

Остальные коэффициенты разложения определяются с помощью разложения Паде [11-14] соотношением типа свертки:

$$
d_{n}=-\sum_{k=0}^{n-1} d_{k} c_{n-k},
$$

которые получаются приравниванием коэффициентов при равных степенях выражения

$$
V(\alpha) / V(\alpha)=1 \text {. }
$$

В развернутом виде (3) имеет вид

$$
\begin{array}{l|l}
\alpha^{0} & d_{0}=c_{0}=1 \\
\alpha & d_{1}=-c_{1} d_{0}=-c_{1} \\
\alpha^{2} & d_{2}=-\left(c_{1} d_{1}+c_{2} d_{0}\right)=c_{1}^{2}-c_{2} \\
\alpha^{3} & d_{3}=-\left(c_{1} d_{2}+c_{2} d_{1}+c_{3} d_{0}\right)=-c_{1}^{3}+2 c_{2} c_{1}-c_{3} \\
\alpha^{4} & d_{4}=-\left(c_{1} d_{3}+c_{2} d_{2}+c_{3} d_{1}+c_{4} d_{0}\right) \\
\alpha^{5} & d_{5}=-\left(c_{1} d_{4}+c_{2} d_{3}+c_{3} d_{2}+c_{4} d_{1}+c_{5} d_{0}\right) \\
\alpha^{6} & d_{6}=-\left(c_{1} d_{5}+c_{2} d_{4}+c_{3} d_{3}+c_{4} d_{2}+c_{5} d_{1}+c_{6} d_{0}\right)
\end{array}
$$

Учитывая дополнительное выражение, получающееся в результате приравнивании коэффициентов при одинаковых степенях в (1)

$$
\begin{gathered}
\left(1+c_{1} \alpha+c_{2} \alpha^{2}+c_{3} \alpha^{3}+c_{4} \alpha^{4}+c_{5} \alpha^{5} \ldots\right)= \\
=1+\alpha \sigma\left(1+d_{1}^{*} \alpha+d_{2}^{*} \alpha^{2}+d_{3}^{*} \alpha^{3}+d_{4}^{*} \alpha^{4}+d_{5}^{*} \alpha^{5} \ldots\right),
\end{gathered}
$$

получаем коэффициенты разложения искомой функции

$$
\begin{gathered}
c_{1}=\sigma, c_{2}=-\sigma \sigma^{*}, c_{3}=\sigma^{2} \sigma^{*}+\sigma \sigma^{* 2}, \\
c_{4}=-\sigma^{3} \sigma^{*}-3 \sigma^{2} \sigma^{* 2}-\sigma \sigma^{* 3}, \\
c_{5}=6 \sigma^{* 3} \sigma^{2}+6 \sigma^{* 2} \sigma^{3}+\sigma^{*} \sigma^{4}+\sigma \sigma^{* 4}, \\
c_{6}=-\sigma \sigma^{* 5}-10 \sigma^{* 4} \sigma^{2}-20 \sigma^{* 3} \sigma^{3}-10 \sigma^{* 2} \sigma^{5}-\sigma^{*} \sigma^{5} .
\end{gathered}
$$

Представим полученные коэффициенты в ряд Тейлора (2)

$$
\begin{gathered}
V(\alpha)=1+c_{1} \alpha+c_{2} \alpha^{2}+c_{3} \alpha^{3}+\ldots= \\
=1+\left(\sigma \alpha-\sigma \sigma^{*}\right) \alpha^{2}+\left(\sigma^{2} \sigma^{*}+\sigma \sigma^{* 2}\right) \alpha^{3}+\ldots
\end{gathered}
$$

Для увеличения радиуса сходимости представим последнее выражение в виде разложения Паде, как отношение двух рациональных функций:

$$
\begin{gathered}
V(\alpha)=[L / M]_{V(\alpha)}=\frac{A(\alpha)}{B(\alpha)}= \\
=\frac{a_{0}+a_{1} \alpha+a_{2} \alpha^{2}+. .+a_{L} \alpha^{L}}{b_{0}+b_{1} \alpha+b_{2} \alpha^{2}+. .+b_{M} \alpha^{M}}
\end{gathered}
$$

числитель $A(\alpha)$ и знаменатель $B(\alpha)$ которого полиномы степени $L$ и $M$ соответственно. Для степеней полиномов выполняется соотношение $L+M=N$. Здесь $N$ - степень исходного полинома $V(\alpha)$. По условию теоремы Шталя $[15,16]$, наилучшая сходимость обеспечивается, если степени полиномов числителя и знаменателя одинаковы, то есть $L=M$. Такой вид разложения Паде называется диагональным. Преобразуем исходное выражение в произведение

$$
\begin{gathered}
V(\alpha) B(\alpha)=A(\alpha) \\
\left(c_{0}+c_{1} \alpha+c_{2} \alpha^{2}+\ldots+c_{M+L} \alpha^{M+L}\right) \times \\
\times\left(b_{0}+b_{1} \alpha+b_{2} \alpha^{2}+\ldots .+b_{M} \alpha^{M}\right)= \\
=a_{0}+a_{1} \alpha+a_{2} \alpha^{2}+\ldots+a_{L} \alpha^{L}
\end{gathered}
$$

и, приравнивая коэффициенты при одинаковых степенях, получаем различные представления диагонального разложения Паде

$$
\begin{gathered}
{[2 / 2]_{V(\alpha)}=\frac{1+\left(2 \sigma+2 \sigma^{*}\right) \alpha+\left(\sigma^{2}+\sigma \sigma^{*}+\sigma^{* 2}\right) \alpha^{2}}{1+\left(\sigma+2 \sigma^{*}\right) \alpha+\sigma^{* 2} \alpha^{2}},} \\
{[3 / 3]_{V(\alpha)}=\frac{\left[\begin{array}{l}
1+\left(3 \sigma+3 \sigma^{*}\right) \alpha+ \\
+\left(\sigma^{2}+\left(\sigma+\sigma^{*}\right)\left(2 \sigma+2 \sigma^{*}\right)+\sigma^{* 2}\right) \alpha^{2}+ \\
+\left(\sigma^{2}+\sigma^{* 2}\right)\left(\sigma+\sigma^{*}\right) \alpha^{3}
\end{array}\right]}{\left[\begin{array}{l}
1+\left(2 \sigma+3 \sigma^{*}\right) \alpha+ \\
+\left[3 \sigma^{* 2}+2 \sigma \sigma^{*}+\sigma^{2}\right] \alpha^{2}+\sigma^{* 3} \alpha^{3}
\end{array}\right]},} \\
{[4 / 4]_{V(\alpha)}=\frac{\left[\begin{array}{l}
1+\left(4 \sigma+4 \sigma^{*}\right) \alpha+\left(6 \sigma^{2}+6 \sigma \sigma^{*}+6 \sigma^{* 2}\right) \alpha^{2}+ \\
+\left(4 \sigma^{3}+6 \sigma^{2} \sigma^{*}+6 \sigma \sigma^{* 2}+4 \sigma^{* 3}\right) \alpha^{3}+ \\
+\left(\sigma^{4}+\sigma^{3} \sigma^{*}+\sigma^{2} \sigma^{* 2}+\sigma \sigma^{* 3}+\sigma^{* 4}\right) \alpha^{4}
\end{array}\right]}{\left[\begin{array}{l}
1+\left(3 \sigma+4 \sigma^{*}\right) \alpha+\left(3 \sigma^{2}+9 \sigma \sigma^{*}+6 \sigma^{* 2}\right) \alpha^{2}+ \\
+\left(\sigma^{3}+2 \sigma^{2} \sigma^{*}+3 \sigma \sigma^{* 2}+4 \sigma^{* 3}\right) \alpha^{3}+\sigma^{* 4} \alpha^{4}
\end{array}\right]}}
\end{gathered}
$$


Приведем числовые значения коэффициентов разложения Паде искомого напряжения $V$ для схемы рис. 3 , если заданы условия в относительных единицах:

$$
\begin{gathered}
Z=R+j X=0,1+j 0,2, \\
S=P+j Q=0,7+j 0,26, U_{1}=1 ; \\
\sigma_{r}=-(R P+X Q)=-0,122, \sigma_{i}=-(R Q-X P)=0,114 .
\end{gathered}
$$

Подставив в разложение Паде вместо $\alpha$ единицу, получим значения искомого напряжения

$$
\begin{aligned}
& {[2 / 2]_{V(\alpha)}=\frac{1-0,488 \alpha+0,031656 \alpha^{2}}{\left[\begin{array}{l}
1+(0,366-j 0,114) \alpha+ \\
+(0,001888-j 0,027816) \alpha^{2}
\end{array}\right]},} \\
& {[2,2]_{V(1)}=0,84721 e^{-j 7,71845} \text {; }} \\
& {[3 / 3]_{V(\alpha)}=\frac{1-0,732 \alpha+0,122848 \alpha^{2}+0,000921344 \alpha^{3}}{\left[\begin{array}{l}
1-(0,61-j 0,114) \alpha+ \\
+(0,063312-j 0,055632) \alpha^{2}+ \\
+(0,002940688+j 0,003608784) \alpha^{3}
\end{array}\right]},} \\
& {[3,3]_{V(1)}=0,84685 e^{-j 7,73564} \text {; }} \\
& {[4 / 4]_{V(\alpha)}=\frac{\left[\begin{array}{l}
1-0,976 \alpha+0,273576 \alpha^{2}+ \\
+0,017290816 \alpha^{3}-0,000657761344 \alpha^{4}
\end{array}\right]}{\left[\begin{array}{l}
1-(0,854-j 0,114) \alpha+ \\
+(0,184272-j 0,083448) \alpha^{2}+ \\
+(0,00230336-j 0,014004672) \alpha^{3}- \\
+(0,000770165312+j 0,000105033216) \alpha^{4}
\end{array}\right]},} \\
& {[4,4]_{V(1)}=0,84683 e^{-j 7,73657} \text {. }}
\end{aligned}
$$

Следует заметить, что различные приближения Паде можно получить, представляя уравнение (1) в виде бесконечной дроби, последовательно подставляя выражение $V(\alpha)$ в себя. Такое представление решения называется представлением Висковатого:

$$
V(\alpha)=1+\frac{\alpha \sigma}{V^{*}(\alpha)}=1+\frac{\alpha \sigma}{1+\frac{\alpha \sigma^{*}}{1+\frac{\alpha \sigma}{1+\frac{\alpha \sigma^{*}}{1+\frac{\alpha \sigma}{1+\frac{\alpha \sigma^{*}}{1+\ldots .}}}}}}
$$

В частности, обрывая бесконечную дробь на 4-й ступени, после упрощения можно получить представление Паде $[2 / 2]_{V(\alpha)}$

$$
\begin{gathered}
V(\alpha)=1+\frac{\alpha \sigma}{1+\frac{\alpha \sigma^{*}}{1+\frac{\alpha \sigma}{1+\alpha \sigma^{*}}}}= \\
=\frac{1+\left(2 \sigma+\sigma^{*}\right) \alpha+\left(\sigma^{2}+\sigma \sigma^{*}+\sigma^{* 2}\right) \alpha^{2}}{1+\left(\sigma+2 \sigma^{*}\right) \alpha+\sigma^{* 2} \alpha^{2}} .
\end{gathered}
$$

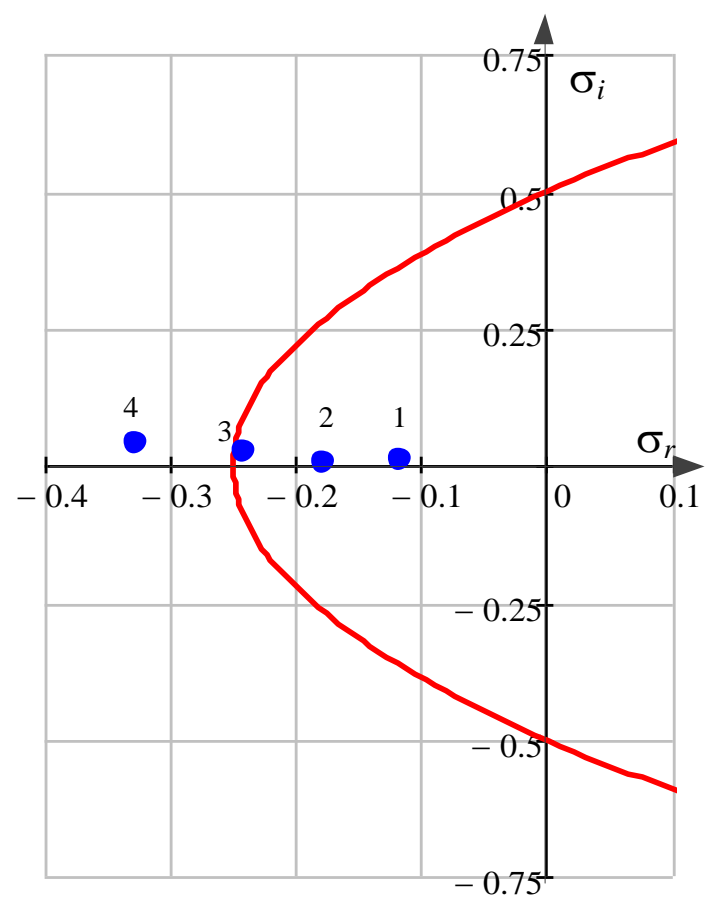

\begin{tabular}{|c|c|c|c|c|c|c|c|}
\hline $\begin{array}{c}\text { Случай/Case } \\
\mathrm{Z}=0,1+j 0,2\end{array}$ & $\begin{array}{c}\text { Нагрузка/Load } \\
P+j Q \text { о.e. }\end{array}$ & $V=|V| e^{j \varphi}$ & $V=[3 / 3]$ & $V=[4 / 4]$ & $V=[6 / 6]$ & $\begin{array}{l}V \text {-Ньютон-Рафсон } \\
\text { Newton-Raphson }\end{array}$ & $\begin{array}{l}\text { Tочное значение } \\
\text { Exact value }\end{array}$ \\
\hline \multirow{2}{*}{1} & \multirow{2}{*}{$0,7+j 0,26$} & $|V|$ & 0,84685 & 0,84683 & $\begin{array}{l}0,84683 \\
\end{array}$ & 0,8495 & 0,8495 \\
\hline & & $\varphi$ & $-7,73564$ & $-7,73657$ & $-7,73662$ & $-7,73662$ & $-7,73662$ \\
\hline \multirow[t]{2}{*}{2} & $0,8+j 0,5$ & $|V|$ & 0,7492 & 0,7488 & 0,7487 & 0,7487 & 0,7487 \\
\hline & & $\varphi$ & $-8,4326$ & $-8,446$ & $-8,448$ & $-8,448$ & $-8,448$ \\
\hline \multicolumn{2}{|c|}{$\begin{array}{c}\text { Граница устойчивости } \\
\text { Stability boundary }\end{array}$} & $|V|$ & 0,4584 & 0,2955 & 0,7712 & \multirow{2}{*}{$\begin{array}{l}\text { Расходится } \\
\text { Diverges }\end{array}$} & 0,5002 \\
\hline 3 & $1,2+j 0,624$ & $\varphi$ & $-20,3427$ & $-25,9667$ & $-9,0324$ & & $-1,7254$ \\
\hline \multicolumn{2}{|c|}{$\begin{array}{l}\text { Нефизические решения } \\
\text { Non-physical solutions }\end{array}$} & $|V|$ & 0,835 & 0,4069 & 0,7237 & \multirow{2}{*}{$\begin{array}{l}\text { Расходится } \\
\text { Diverges }\end{array}$} & 0,7524 \\
\hline 4 & $1,5+j 0,9$ & $\varphi$ & $-5,8181$ & $\begin{array}{l}-19,853 \\
\end{array}$ & \begin{tabular}{|l|}
$-9,6968$ \\
\end{tabular} & & $-48,3552$ \\
\hline
\end{tabular}

Рис. 3. Пример расположения точек из табл. 1 относительно границы устойчивости

Fig. 3. Example of arrangement of points from table 1 relative to the stability boundary

\section{Сравнение результатов методов}

Сведем в табл. 1 все рассчитанные значения искомого $V$ напряжения при различном разложении Паде, методом Ньютона-Рафсона и аналитическим методом.

Таблица 1. Результаты расчета коэффициентов степенных рядов

Table 1. Results of calculation of power series coefficients 


\section{Пример использования метода голоморфного} погружения для трехузловой схемь

Приведем пример использования метода ГП для трехузловой схемы (рис. 4). В представленной схеме узел 1 - балансирующий узел, узлы 2 и 3 - узлы $P Q$ типа. Мощность нагрузок равна $S_{2}=50+j 15 M B \cdot A$ и $S_{3}=45+j 25 M B \cdot A$. Сопротивления ветвей равны $Z_{12}=0,03+j 0,13$ o.e., $Z_{13}=0,01+j 0,08$ o.e., $Z_{23}=0,05+j 0,2$ о.е. Проводимости на землю $Y_{c 12}=Y_{c 13}=Y_{c 23}=j 0,01$ o.e. Расчет будем вести в относительных единицах, считая, что базисная мощность $S_{\mathrm{5}}=100 M B \cdot A$.

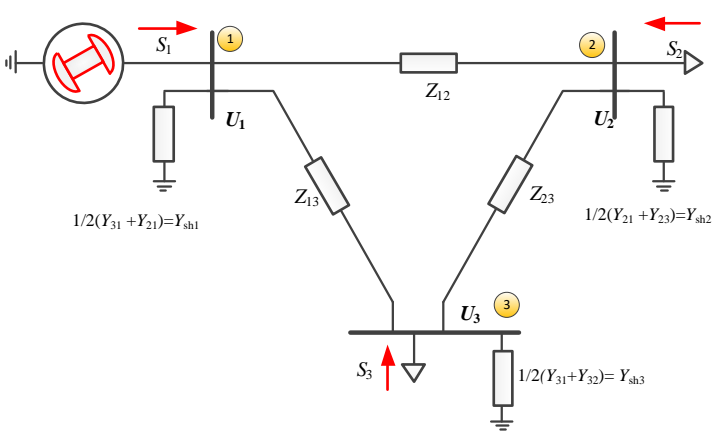

Рис. 4. Трехузловая однолинейная схема электропередачи Fig. 4. Three bus one-line transmission system diagram

Запишем общее узловое уравнение в форме баланса токов для узла $P Q$-типа:

$$
\sum_{j=1}^{n} Y_{i j} U_{j}=\frac{S_{i}^{*}}{U_{i}^{*}},
$$

где $Y_{i j}$ - элементы матрицы узловых проводимостей; $S_{i}=S_{\Gamma i}-S_{\text {н } i}-$ результирующая комплексная мощность, входящая в $i$-й узел; $\dot{U}_{i}-$ напряжение узла $i$. Левую часть равенства (4) можно представить в следующем виде [17-19]:

$$
\sum_{j=1}^{n} Y_{i j} U_{j}=\sum_{j=1}^{n} Y_{i j t r} U_{j}+Y_{i s h} U_{i},
$$

где $\underline{Y}_{i s h}$ - суммарная проводимость на землю узла $I$; $\underline{Y}_{i j t r}$ - элементы матрицы узловых проводимостей без учета проводимостей на землю в диагональных элементах. Переносим слагаемое с проводимостями на землю в выражении (5), в правую часть равенства и осуществляем голоморфное погружение уравнения в комплексную плоскость путем встраивания комплексного параметра $\alpha$. В результате получается следующее выражение [17-19]:

$$
\sum_{j=1}^{n} Y_{i j t r} U_{j}(\alpha)=\frac{\alpha S_{i}^{*}}{U_{i}^{*}\left(\alpha^{*}\right)}-\alpha Y_{i s h} U_{i}(\alpha) .
$$

Так как теперь напряжения являются голоморфными функциями, зависящими от комплексной переменной $\alpha$, их можно разложить в ряд Тейлора

$$
\begin{aligned}
& U_{j}(\alpha)=U_{j}[0]+U_{j}[1] \alpha+U_{j}[2] \alpha^{2}+\ldots+U_{j}[n] \alpha^{n}, \\
& U_{j}^{*}\left(\alpha^{*}\right)=U_{j}^{*}[0]+U_{j}^{*}[1] \alpha+U_{j}^{*}[2] \alpha^{2}+\ldots+U_{j}^{*}[n] \alpha^{n} .
\end{aligned}
$$

Подставим (7) и (8) в равенство (6), получим следующее выражение

$$
\begin{gathered}
\sum_{j=1}^{n} Y_{i j t r}\left(U_{j}[0]+U_{j}[1] \alpha+U_{j}[2] \alpha^{2}+\ldots+U_{j}[n] \alpha^{n}\right)= \\
=\frac{\alpha S_{i}^{*}}{U_{i}^{*}[0]+U_{i}^{*}[1] \alpha+U_{i}^{*}[2] \alpha^{2}+\ldots+U_{i}^{*}[n] \alpha^{n}}- \\
-\alpha Y_{i s h}\left(U_{i}[0]+U_{i}[1] \alpha+U_{i}[2] \alpha^{2}+\ldots+U_{i}[n] \alpha^{n}\right) .
\end{gathered}
$$

Задача сводится к нахождению коэффициентов ряда, удовлетворяющих уравнению (6). Для решения задачи введем функцию $W^{*}\left(\alpha^{*}\right)$ обратную $\dot{U}_{i}^{*}\left(\alpha^{*}\right)$, которую также можно разложить в степенной ряд:

$$
\begin{gathered}
W^{*}\left(\alpha^{*}\right)=\frac{1}{U^{*}\left(\alpha^{*}\right)}= \\
=W^{*}[0]+W^{*}[1] \alpha+W^{*}[2] \alpha^{2}+\ldots+W^{*}[n] \alpha^{n} .
\end{gathered}
$$

Подставим (10) в (9) и получим

$$
\begin{gathered}
\sum_{j=1}^{n} Y_{i j t r}\left(U_{j}[0]+U_{j}[1] \alpha+U_{j}[2] \alpha^{2}+\ldots+U_{j}[n] \alpha^{n}\right)= \\
=\alpha S_{i}^{*}\left(W_{i}^{*}[0]+W_{i}^{*}[1] \alpha+W_{i}^{*}[2] \alpha^{2}+\ldots+W_{i}^{*}[n] \alpha^{n}\right)- \\
-\alpha Y_{i s h}\left(U_{i}[0]+U_{i}[1] \alpha+U_{i}[2] \alpha^{2}+\ldots+U_{i}[n] \alpha^{n}\right) .
\end{gathered}
$$

Для того чтобы определить неизвестные коэффициенты функции $W(\alpha)$, воспользуемся равенством $W^{*}\left(\alpha^{*}\right) U^{*}\left(\alpha^{*}\right)=1$ или $W(\alpha) U(\alpha)=1$. Распишем функции, входящие в данное равенство

$$
\begin{gathered}
\left(W[0]+W[1] \alpha+W[2] \alpha^{2}+\ldots+W[n] \alpha^{n}\right) \times \\
\times\left(U[0]+U[1] \alpha+U[2] \alpha^{2}+\ldots+U[n] \alpha^{n}\right)=1 .
\end{gathered}
$$

В правой части равенства (12) открываем скобки и, приравнивая коэффициенты при одинаковых степенях с обеих сторон уравнения, получаем

$$
\begin{aligned}
& W[0] \cdot U[0]=1 \Rightarrow \\
& \quad \Rightarrow W[0]=\frac{1}{U[0]} ; \\
& W[0] \cdot U[1]+W[1] \cdot U[0]=0 \Rightarrow \\
& \quad \Rightarrow W[1]=-\frac{W[0] \cdot U[1]}{U[0]} ; \\
& W[0] \cdot U[2]+W[1] \cdot U[1]+W[2] \cdot U[0]=0 \Rightarrow \\
& \quad \Rightarrow W[2]=-\frac{W[0] \cdot U[2]+W[1] \cdot U[1]}{U[0]} .
\end{aligned}
$$

Анализируя полученные уравнения, можно записать общее выражение для вычисления $n$-го коэффициента ряда функции $W(\alpha)$ для каждого $n \geq 1$ :

$$
\dot{W}[n]=-\frac{\sum_{k=0}^{n-1} \dot{W}[k] \dot{U}[n-k]}{\dot{U}[0]}, n \geq 1 .
$$

Таким образом, для узла $P Q$-типа неизвестный $n$-й коэффициент функции напряжения $U(\alpha)$ может быть найден по известным (n-1)-м коэффициентам по выражению

$$
\sum_{j=1}^{n} Y_{i j t r} U_{j}[n]=S_{i}^{*} W_{i}^{*}[n-1]-Y_{i s h} U_{i}[n-1] .
$$

Выражение (11), (14) можно представить в матричном виде 


$$
\left[\mathbf{Y}_{\mathrm{ij} \mathrm{tr}}\right] \mathrm{U}[\mathbf{n}]=\mathbf{S}^{*} \mathbf{W}^{*}[\mathbf{n}-\mathbf{1}]-\mathbf{Y}_{\mathrm{sh}} \mathrm{U}[\mathbf{n}-\mathbf{1}] .
$$

Здесь $\left[\underline{\mathbf{Y}}_{\mathrm{ij} \text { tr}}\right]-$ матрица узловых проводимостей без учета проводимостей на землю в диагональных элементах и с учетом балансирующего узла; $\mathbf{U}[\mathbf{n}]-$ вектор-столбец $n$-х коэффициентов степенных функций $U_{i}(\alpha)$ напряжений в узлах; $\mathbf{S}^{*}$ - вектор-столбец задающих мощностей в узлах; $\mathbf{W}^{*}[\mathbf{n}-1]-$ векторстолбец $(n-1)$-х коэффициентов степенных функций $W_{i}(\alpha)$, обратных функциям напряжений в узлах; $\mathbf{Y}_{\mathbf{s h}}-$ вектор-столбец суммарных проводимостей на землю.

Для удобства уравнение (15) представим в виде

$$
\left[\mathbf{Y}_{\mathrm{ij} \mathrm{tr}}\right] \mathbf{U}[\mathbf{n}]=\mathbf{I}[\mathbf{n}-\mathbf{1}],
$$

где $\mathbf{I}[\mathbf{n}-\mathbf{1}]=\mathbf{S}^{*} \mathbf{W}^{*}[\mathbf{n}-\mathbf{1}]-\mathbf{Y}_{\text {sh }} \mathbf{U}[\mathbf{n}-\mathbf{1}]$ - вектор-столбец $(n-$ 1)-х коэффициентов задающих токов в узлах.

Формируем матрицу узловых проводимостей

$$
Y=\left(\begin{array}{ccc}
3,224-j 19,601 & -1,685+j 7,303 & -1,538+j 12,308 \\
-1,685+j 7,303 & 2,862-j 11,999 & -1,176+j 4,706 \\
-1,538+j 12,308 & -1,176+j 4,706 & 2,715-j 17,004
\end{array}\right) .
$$

Рассчитываем матрицу задающих мощностей в узлах

$$
S=\left(\begin{array}{c}
0 \\
-0,5-j 0,15 \\
-0,45-j 0,25
\end{array}\right) \text {. }
$$

Рассчитываем матрицу суммарных проводимостей на землю

$$
Y_{s h}=\left(\begin{array}{c}
j 0,01 \\
j 0,01 \\
j 0,01
\end{array}\right)
$$

Формируем матрицу узловых проводимостей без учета проводимостей на землю в диагональных элементах с учетом того, что узел 1 является балансирующим

$$
Y_{t r}=\left(\begin{array}{ccc}
1 & 0 & 0 \\
-1,685+j 7,303 & 2,862-j 12,009 & -1,176+j 4,706 \\
-1,538+j 12,308 & -1,176+j 4,706 & 2.715-j 17,014
\end{array}\right) .
$$

Начальное значение (0-й коэффициент) напряжений в узлах принимаем равным единице

$$
U[0]=\left(\begin{array}{l}
1 \\
1 \\
1
\end{array}\right)
$$

Тогда 0-е коэффициенты функций $W_{i}(\alpha)$, обратных функциям напряжений в узлах,

$$
W[0]=\frac{1}{U[0]}=\left(\begin{array}{l}
1 \\
1 \\
1
\end{array}\right) .
$$

Находим вектор-столбец задающих токов в узлах при $n=0$

$$
I[0]=\left(\begin{array}{c}
0 \\
-0,5+j 0,14 \\
-0,45+j 0,24
\end{array}\right) \text {. }
$$

Рассчитаем вектор-столбец 1-х коэффициентов степенных функций напряжений $U(\alpha)$ в узлах

$$
U[1]=\left[\mathbf{Y}_{\mathrm{ij} \mathrm{tr}}\right]^{-1} \mathbf{I}[0]=\left(\begin{array}{c}
0 \\
-0,0301-j 0,0522 \\
-0,025-j 0,039
\end{array}\right) .
$$

Используя формулу (10), рассчитываем матрицу 1-х коэффициентов функций $W(\alpha)$, обратных функциям напряжений $U(\alpha)$ в узлах

$$
W[1]=\left(\begin{array}{c}
0 \\
0,03+j 0,052 \\
0,025+j 0,039
\end{array}\right) .
$$

Аналогично рассчитываем следующие коэффициенты, используя (13). Достаточная точность $\varepsilon=10^{-5}$ достигнута после расчета 4-го коэффициента степенного ряд. Результаты расчета представлены в табл. 2.

Таблица 2. Результаты расчета коэффициентов степенных рядов напряжений. Напряжения приведены в относительных единицах

Table 2. Results of calculation of voltage power series coefficients. Voltages are given in relative units

\begin{tabular}{|c|c|c|c|}
\hline $\mathrm{n}$ & $\mathrm{U}_{1}[\mathrm{n}]$ & $\mathrm{U}_{2}[\mathrm{n}]$ & $\mathrm{U}_{3}[\mathrm{n}]$ \\
\hline 0 & 1 & 1 & 1 \\
\hline 1 & 0 & $-0,03-\mathrm{j} 0,052$ & $-0,025-\mathrm{j} 0,039$ \\
\hline 2 & 0 & $-3,516 \cdot 10^{-3}-\mathrm{j} 3,378 \cdot 10^{-5}$ & $-2,381 \cdot 10^{-3}-\mathrm{j} 1,496 \cdot 10^{-5}$ \\
\hline 3 & 0 & $-2,07 \cdot 10^{-4}-\mathrm{j} 4,097 \cdot 10^{-6}$ & $-1,325 \cdot 10^{-4}-\mathrm{j} 2,966 \cdot 10^{-6}$ \\
\hline 4 & 0 & $-2,324 \cdot 10^{-5}-\mathrm{j} 3,866 \cdot 10^{-7}$ & $-1,42 \cdot 10^{-5}-\mathrm{j} 3,562 \cdot 10^{-7}$ \\
\hline
\end{tabular}

Таким образом, функции напряжений во втором и третьем узлах, представленные в виде степенного ряда, имеют следующий вид

$$
\begin{aligned}
& U_{2}(\alpha)=1+(-0,03-j 0,052) \alpha+ \\
& +\left(-3,516 \cdot 10^{-3}-j 3,378 \cdot 10^{-5}\right) \alpha^{2}+ \\
& +\left(-2,07 \cdot 10^{-4}+j 4,097 \cdot 10^{-6}\right) \alpha^{3}+ \\
& +\left(-2,324 \cdot 10^{-5}+j 3,866 \cdot 10^{-7}\right) \alpha^{4} ; \\
& U_{3}(\alpha)=1+(-0,025+j 0,039) \alpha+ \\
& +\left(-2,381 \cdot 10^{-3}+j 1,496 \cdot 10^{-5}\right) \alpha^{2}+ \\
& +\left(-1,325 \cdot 10^{-4}-j 2,966 \cdot 10^{-6}\right) \alpha^{3}+ \\
& +\left(-1,42 \cdot 10^{-5}-j 3,562 \cdot 10^{-7}\right) \alpha^{4} .
\end{aligned}
$$

Данные функции могут быть представлены в виде аппроксимантов Паде порядка [2/2]

$$
\begin{gathered}
U_{2}(\alpha)= \\
=\frac{1+(-0,118-j 0,0031) \alpha+(0,000172+j 0,000212) \alpha^{2}}{1+(-0,0879+j 0,0491) \alpha+(-0,00152-j 0,00287) \alpha^{2}} ; \\
U_{3}(\alpha)= \\
=\frac{1+(-0,109+j 0,0052) \alpha+(0,000259-j 0,00031) \alpha^{2}}{1+(-0,0844+j 0,0442) \alpha+(-0,00119-j 0,00251) \alpha^{2}} .
\end{gathered}
$$

Подставляя значение $\alpha=1$, получаем искомое решение

$$
\begin{gathered}
U_{2}(1)=0,9662+j 0,0522=0,9676 e^{-j 3,094^{\circ}} \text { o.e., } \\
U_{3}(1)=0,9724+j 0,039=0,9732 e^{-j 2,294^{\circ}} \text { o.e. }
\end{gathered}
$$

Результаты расчета совпадают с результатами расчета методом Ньютона-Рафсона, полученными в ПК «RastrWin3» и MATPOWER (табл. 3). 
Таблица 3. Сравнение результатов расчета. Напряжения приведены в относительных единицах. $U=V e^{j \delta}$

Table 3. Comparison of calculation results. Voltages are given in relative units. $U=V e^{j \delta}$

\begin{tabular}{|c|c|c|c|c|c|c|}
\hline \multirow[t]{2}{*}{ 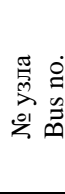 } & \multicolumn{2}{|c|}{$\begin{array}{c}\text { Метод голоморф- } \\
\text { ного погружения } \\
\text { Holomorphic } \\
\text { embedding method }\end{array}$} & \multicolumn{2}{|c|}{ RastrWin3 } & \multicolumn{2}{|c|}{ MATPOWER } \\
\hline & V & $\begin{array}{c}\delta, \\
\text { градусы } \\
\text { degree }\end{array}$ & V & $\begin{array}{c}\delta, \text { градусы } \\
\text { degree }\end{array}$ & V & $\delta, \begin{array}{c}\text { градусь } \\
\text { degree }\end{array}$ \\
\hline 1 & 1,0000 & 0,0000 & 1,0000 & 0,0000 & 1,0000 & 0,0000 \\
\hline 2 & 0,9676 & $-3,0936$ & 0,9676 & $-3,0936$ & 0,9676 & $-3,0936$ \\
\hline 3 & 0,9732 & $-2,2942$ & 0,9732 & $-2,2942$ & 0,9732 & $-2,2942$ \\
\hline
\end{tabular}

\section{Заключение}

В работе представлен аналитический метод ГП, обладающий рядом преимуществ перед известным методом Ньютона-Рафсона:

- физической наглядностью;

- простотой алгоритмической реализации, заключающейся в рекуррентных соотношениях для коэффициентов разложения искомой функции в ряд Тейлора;

- разложенная в ряд функция является голоморфной, что позволяет осуществлять ее аналитическое продолжение, аналитическое продолжение позволяет получить желаемую точность.

Авторы работы привели примеры использования метода ГП для двух- и трехузловых схем.

К недостаткам метода следует отнести:

- $\quad$ неудовлетворительную работу при приближении к границе устойчивости за счет появления ложных нулей знаменателя в разложении Паде искомого решения. При приближении к границе устойчивости алгебраические уравнения для определения коэффициентов разложения решения становятся

\section{СПИСОК ЛИТЕРАТУРЫ}

1. Новые технологии и современное оборудование в энергетике нефтегазовой промышленности / И.В. Белоусенко, Г.Р. Шварц С.Н. Великий, М.С. Ершов, А.Д. Яризов. - М.: ООО «НедраБизнесцентра», 2007. $-478 \mathrm{c}$.

2. Архипова О.В., Ковалев В.З., Хамитов Р.Н. Методика моделирования регионально обособленного электротехнического комплекса // Известия Томского политехнического университета. Инжиниринг георесурсов. - 2019. - Т. 330. - № 1. C. $173-180$.

3. Идельчик В.И. Расчеты установившихся режимов электрических систем. - М.: Энергия, 1977. - 192 с.

4. Вычислительные модели потокораспределения в электрических системах / Б.И. Аюев, В.В. Давыдов, П.М. Ерохин, В.Г. Неуймин. - М.: Изд-во «Флинта», 2008. - 256 с.

5. Тарасов В.И. Теоретические основы анализа установившихся режимов электроэнергетических систем. - Новосибирск: Наука, 2002. - 344 с.

6. Жуков Л.А., Стратан И.П. Установившиеся режимы сложных электрических сетей и систем: методы расчетов. - М.: Энергия, 1979. - $416 \mathrm{c.}$

7. Гуревич Ю.Е., Либова Л.Е., Окин А.А. Расчеты устойчивости и противоаварийной автоматики в энергосистемах. - М.: Энергоатомиздат, 1990. - 390 с.

8. Trias A. Fundamentals of the holomorphic embedding load-flow method. URL: https://arxiv.org/abs/1509.02421 (дата обращения: 15.01.2020)

9. Шабат Б.В. Введение в комплексный анализ. Ч. 1: Функции одного переменного. - М.: Наука, 2004. - 336 с. плохо обусловленными. Поэтому следует применять методы регуляризации решения.

В последнее время появились работы, позволяющие получать аналитические решения на границе устойчивости [20]. Это происходит за счет дополнительного пересчета решения, с новыми стартовыми значениями, форсирующего процесс схождения к решению и позволяющего осуществлять аналитическое продолжение вдоль особым образом выбранного выреза в комплексной плоскости.

- отсутствие четкого критерия приближения решения к границе устойчивости.

Существуют работы, в которых предлагается формировать критерий приближения к границе устойчивости на основе расположения нулей и полюсов разложения Паде на корневом годографе [21, 22]. Корни разложения решения в ряд Тейлора располагаются по окружности, радиус которой определяется критерием Каши-Адамара.

$$
\frac{1}{R}=\lim _{n \rightarrow \infty}\left|c_{n}\right|^{1 / n} \quad V(\alpha)=1+c_{1} \alpha+c_{2} \alpha^{2}+\ldots+c_{n} \alpha^{n} .
$$

По отклонению корней и полюсов разложения Паде от действительной оси можно судить о наличии ложных корней в разложении, а следовательно, и о сходимости решения. Если решения приближаются к границе устойчивости, то корни приближаются к окружности радиуса, определяемого критерием Фабри с внутренней стороны $[21,22]$

$$
R=\lim _{n \rightarrow \infty} \frac{\left|c_{n}\right|}{\left|c_{n+1}\right|}, V(\alpha)=1+c_{1} \alpha+c_{2} \alpha^{2}+\ldots+c_{n} \alpha^{n} .
$$

Таким образом, приближение корней к окружности радиуса $R$ может служить индикатором приближения решения к границе устойчивости.

10. Murduck J.A. Perturbation theory and methods. - New York: John Wiley \& Sons, Inc, 1991. -509 p.

11. Суетин С.П. Аппроксимации Паде и эффективное аналитическое продолжение степенного ряда // УМН. - 2002. - Т. 57. Вып. 1 (343). - С. 45-142.

12. Бейкер Дж. мл., Грейвс-Моррис П. Аппроксимация Паде. - М.: Мир, 1986. -502 c.

13. Никишин Е.М., Сорокин В.Н. Рациональные аппроксимации и ортогональность. - М.: Наука, 1988. -258 с.

14. Trias A. The holomorphic embedding load flow method // Power and Energy Society General Meeting. - San Diego, July 2012. - P. 1-8.

15. Stahl H. Orthogonal polynomials with complex-valued function. I, II // Constr. Approx. - 1986. - V. 2. - P. 225-251.

16. Stahl H. Domains associated with an analitic function. I // Complex Variables Theory Appl. - 1985. - V. 4 (4). - P. 311-324.

17. Online voltage stability assessment for load areas based on the holomorphic embedding method / Chengxi Liu, Bin Wang, Fengkai Hu, Kai Sun, Claus Leth Bak // IEEE Transactions on Power Systems. - 2018. - V. 33. - № 4. - P. 3720-3734.

18. Chiang H., Wang T., Sheng H. A novel fast and flexible holomorphic embedding power flow method // IEEE Transactions on Power Systems. - 2018. - V. 33. - № 3. - P. 2551-2562.

19. Feng Y., Tylavsky D. A holomorphic embedding approach for finding the Type-1 power-flow solutions // International Journal of Electrical Power \& Energy Systems. - 2018. - V. 102. - P. 179-188.

20. Rao S.D., Tylavsky D.J., Feng Y. Estimating the saddle-node bifurcation point of static power systems using the holomorphic embedding method // International Journal of Electrical Power \& Energy Systems. - 2017. - V. 84. - P. 1-12. 
Известия Томского политехнического университета. Инжиниринг георесурсов. 2020. Т. 331. № 4. 115-125

Исаев Ю.Н., Кабалин Д.А. Голоморфное погружение как аналитический метод расчета электрических сетей нефртяных и ...

21. Suetin S.P., Baghsorkhi S.S. Embedding AC Power Flow in the Complex Plane. Part I: Modelling and Mathematical Foundation. 2016. - 13 p. URL: https://arxiv.org/abs/1604.03425 (дата обращения 15.01.2020).
22. Suetin S.P., Baghsorkhi S.S. Embedding AC Power Flow in the Complex Plane. Part II: A Reliable Framework for Voltage Collapse Analysis. - 2016. URL: https://arxiv.org/abs/1604.03425 (дата обращения 15.01.2020).

Поступила: 24.01.2020 г.

\section{Информация об авторах}

Исаев Ю.Н., доктор физико-математических наук, профессор отделения электроэнергетики и электротехники Инженерной школы энергетики Национального исследовательского Томского политехнического университета.

Кабалин Д.А., аспирант Инженерной школы энергетики Национального исследовательского Томского политехнического университета. 
UDC 621.314.58:681.5.015

\title{
HOLOMORPHIC EMBEDDING AS ANALYTICAL TECHNIQUE FOR CALCULATING ELECTRIC GRIDS OF OIL AND GAS DEPOSITS
}

\author{
Yusup N. Isaev', \\ isaev_yusup@mail.ru \\ Dmitry A. Kabalin 1 , \\ mitya.kabalin@gmail.com \\ 1 National Research Tomsk Polytechnic University, \\ 30, Lenin avenue, Tomsk, 634050, Russia.
}

In order to ensure the required level of reliable operation of oil production, it is necessary to pay attention to the operating conditions of the power system. This is important if we take into account that for oil and gas extraction the powerful sources of energy that significantly affect the mode of operation of the power grid are needed. In this case, the urgent task is to calculate the steady-state modes of the oil production electric network. The calculations of the established modes are of great practical importance to ensure efficient and safe management of the operating modes of oil and gas enterprises, and are important in the design of electrical networks for oil and gas enterprises. However, the application of classical iterative methods for calculating steady-state regimes, such as the Gauss-Seidel and Newton-Raphson methods, does not always allow finding the right solution, since the convergence of these methods depends on initial conditions. The method is based on the Padé approximation and the perturbation method. The paper demonstrates the disadvantages and the advantages of the proposed method over the well-known Gauss-Seidel and Newton-Raphson iteration method and the examples of solving the problems of electric power chains. The problems of sustainability are considered.

The aim of the research is to apply the analytical method of holomorphic embedding to calculate two and three nodal energy schemes; compare the capabilities of the method with other alternative methods; investigate the limitations of the holomorphic embedding method and show the area of its work.

Methods: Taylor expansion, analytic continuation, solving algebraic equations by the recurrent method, infinite fractions.

Results. The authors gave the examples of using the holomorphic embedding method for two and three PQ nodal circuits, and showed the shortcomings of the holomorphic embedding method. The holomorphic embedding method is compared with alternative methods.

Conclusions. The analytical method of holomorphic embedding has several advantages: physical visibility; the simplicity of the algorithmic implementation consisting in recurrence relations for the coefficients of the expansion of the desired function in a Taylor series. The function laid out in a series is holomorphic, which allows analytic continuation of a function to obtain the desired accuracy of solution.

\section{Key words:}

Energy system, steady process, stability, nonlinear equations, holomorphic e, Padé decomposition, convergence.

\section{REFERENCES}

1. Belousenko I.V., Schwartz G.R., Great S.N., Ershov M.S. Yarizov A.D. Novye tekhnologii i sovremennoe oborudovanie v energetike neftegazovoy promyshlennosti [New technologies and modern equipment in the energy sector of the oil and gas industry]. Moscow, Nedra-Biznestsentra Publ., 2007. 478 p.

2. Arkhipova O.V., Kovalev V.Z., Khamitov R.N. Methodology of modeling regionally isolated electrotechnical complex. Bulletin of the Tomsk Polytechnic University. Geo Assets Engineering, 2019, vol. 330, no. 1, pp. 173-180. In Rus.

3. Idelchik V.I. Raschety ustanovivshikhsya rezhimov elektricheskikh sistem [Calculations of steady-state modes of electric systems] Moscow, Energia Publ., 1977. 192 p.

4. Ayuev B.I., Davydov V.V., Erokhin P.M., Neuiymin V.G. Vychislitelnye modeli potokoraspredeleniya $v$ elektricheskikh sistemakh [Power flow computing models in power systems]. Moscow, Nauka Publ., 2008. 256 p.

5. Tarasov V.I. Teoreticheskie osnovy analiza ustanovivshikhsya rezhimov elektroenergeticheskikh sistem [Theoretical bases of analysis of steady-state modes of electric power systems]. Novosibirsk, Nauka Publ., 2002. 344 p.

6. Zhukov L.A., Stratan I.P. Ustanovivshiesya rezhimy slozhnykh elektricheskikh setey $i$ sistem: metody raschetov [Steady-state modes of complex electrical networks and systems: methods of calculations]. Moscow, Energia Publ., 1979. 416 p.

7. Gurevich Yu.E., Libova L.E., Okin A.A. Raschety ustoychivosti $i$ protivoavariynoy avtomatiki $v$ energosistemakh [Calculation of stability and emergency automation in power systems]. Moscow, Energoatomizdat Publ., 1990. 390 p.

8. Trias A. Fundamentals of the holomorphic embedding load-flow method. Available at: https://arxiv.org/abs/1509.02421 (accessed 15 Janyuary 2020).
9. Shabat B.V. Vvedenie v kompleksny analiz. Ch. 1: Funktsii odnogo peremennogo [Introduction to complex analysis. P. 1: Functions of one variable]. Moscow, Nauka Publ., 2004. 336 p.

10. Murduck J.A. Perturbation theory and methods. New York, John Wiley \& Sons, Inc, $1991.509 \mathrm{p}$.

11. Suetin S.P. Padé approximants and the effective analytic continuation of a power series. Russian Math. Surveys, 2002, vol. 57, no. 1, pp. 45-142.

12. Baker G.A., Graves-Morris P. Approksimatsia Pade [Padé approximants]. Moscow, Mir Publ., 1986. 502 p.

13. Nikishin E.M., Sorokin V.N. Ratsionalnye approksimatsii $i$ ortogonalnost [Rational approximations and orthogonality]. Moscow, Nauka Publ., 1988. 258 p.

14. Trias A. The holomorphic embedding load flow method. Power and Energy Society General Meeting. San Diego, July 2012. pp. 1-8.

15. Stahl H. Orthogonal polynomials with complex-valued function. I, II. Constr. Approx., 1986, vol. 2, pp. 225-251.

16. Stahl H. Domains associated with an analitic function. I. Complex Variables Theory Appl., 1985, vol. 4 (4), pp. 311-324.

17. Chengxi Liu, Bin Wang, Fengkai Hu, Kai Sun, Claus Leth Bak. Online voltage stability assessment for load areas based on the holomorphic embedding method. IEEE Transactions on Power Systems, 2018, vol. 33, no. 4, pp. 3720-3734.

18. Chiang H., Wang T., Sheng H. A novel fast and flexible holomorphic embedding power flow method. IEEE Transactions on Power Systems, 2018, vol. 33, no. 3, pp. 2551-2562.

19. Feng Y., Tylavsky D. A holomorphic embedding approach for finding the Type-1 power-flow solutions. International Journal of Electrical Power \& Energy Systems, 2018, vol. 102, pp. 179-188.

20. Rao S.D., Tylavsky D.J., Feng Y. Estimating the saddle-node bifurcation point of static power systems using the holomorphic 
embedding method. International Journal of Electrical Power \& Energy Systems, 2017, vol. 84, pp. 1-12.

21. Suetin S.P., Baghsorkhi S.S. Embedding AC Power Flow in the Complex Plane. P. I: Modelling and Mathematical Foundation, 2016. 13 p. Available at: https://arxiv.org/abs/1604.03425 (accessed 15 January 2020).
22. Suetin S.P., Baghsorkhi S.S. Embedding AC Power Flow in the Complex Plane. P. II: A Reliable Framework for Voltage Collapse Analysis. 2016. Available at: https://arxiv.org/abs/1604.03425 (accessed 15 January 2020).

\section{Information about the authors}

Yusup N. Isaev, Dr. Sc., professor, National Research Tomsk Polytechnic University.

Dmitry A. Kabalin, postgraduate student, National Research Tomsk Polytechnic University. 